\title{
Resposta diferencial de linhagens de feijoeiro ao nitrogênio
}

\author{
Differential response of breeding lines of common bean to nitrogen
}

\author{
Isabela Volpi Furtini' ${ }^{1}$ MagnoAntonio Patto Ramalho \\ Ângela de Fátima Barbosa $\mathrm{Abreu}^{3}$ \\ Antonio Eduardo Furtini Neto ${ }^{4}$
}

\section{RESUMO}

Visando à identificação de linhagens de feijão que sejam tolerantes ao estresse de nitrogênio $(N)$ e que também apresentem resposta positiva a esse nutriente, foram avaliadas 100 linhagens em presença e ausência de $N$ em cobertura, em quatro ambientes. Em cada ambiente, foram dois experimentos distintos com e sem $N$, no delineamento látice triplo 10x10, sendo a parcela constituída de uma linha de três metros de comprimento. Utilizando a produtividade média de grãos, foi estimado o índice de eficiência de utilização de nitrogênio. $\mathrm{Na}$ média dos quatro ambientes, a produtividade de grãos obtida com $N$ foi $12 \%$ acima da obtida sem N. Decompondo o efeito de níveis para cada linhagem, em $77 \%$ delas não houve resposta à adubação nitrogenada em cobertura. Assim, apenas 22 linhagens responderam positivamente à adubação nitrogenada e, entre elas, a eficiência na utilização de $N$ variou de 11,3 a 18,3kg de grãos por $\mathrm{kg}$ de $\mathrm{N}$ aplicado.

Palavras-chave: eficiência do uso de nitrogênio, melhoramento, genético, Phaseolus vulgaris $\mathrm{L}$.

\section{ABSTRACT}

It was evaluated 100 breeding lines of common bean in the presence and absence of $N$ in cover in four conditions aiming at identifing the lines of beans which would be tolerant to nitrogen $(N)$ stress and/or also responsive to this nutrient. In each condition there were two distinct experiments with and without $N$, in a $10 \times 10$ triple lattice design, being the plot constituted by a line with three meters of length. It was estimated the index of efficiency of nitrogen utilization, through the average grains yield. The obtained yield with $N$ was $12 \%$ above the one without $N$, in the average of the four conditions. By decomposing the effect of levels for each line, in $77 \%$ of them there was no answer to the nitrogen fertilization in cover. This, only 22 lines positively responded to nitrogen fertilization and among them the efficiency of $N$ utilization ranged from 11.3 to $18.3 \mathrm{~kg}$ of grains per $\mathrm{kg}$ of applied $N$.

Key words: nitrogen use efficiency, improvement, genetic, Phaseolus vulgaris $L$.

\section{INTRODUÇÃO}

A fixação de nitrogênio (N) por microrganismos do gênero Rhizobium (FBN) na cultura do feijoeiro (Phaseolus vulgaris L.) vem recebendo atenção da pesquisa ao longo do tempo. Embora se tenha obtido considerável avanço no conhecimento dos fatores que afetam a FBN, há inúmeros trabalhos que mostram que a inoculação com Rhizobium não fornece o nitrogênio que a cultura necessita para atingir níveis econômicos de produtividade de grãos (ALVES, 2002; SILVA, 2002; FERREIRA et al., 2004). Por essa razão, entre os insumos que mais contribuem para a produtividade de grãos, está o emprego de fertilizantes nitrogenados. Há disparidade muito grande entre os agricultores na utilização de N. A grande maioria, que cultiva pequenas áreas, em agricultura tipicamente familiar, praticamente não o utiliza. No outro extremo, os empresários rurais, que cultivam extensas áreas irrigadas, empregam enorme quantidade desse nutriente, normalmente doses superiores a $100 \mathrm{~kg} \mathrm{ha}^{-1}$ (FERREIRA et al., 2004).

${ }^{1}$ Curso de Agronomia, Universidade Federal de Lavras (UFLA), Lavras, MG, Brasil.

${ }^{2}$ Departamento de Biologia, UFLA, CP 3037, 37200-000, Lavras, Minas Gerais, Brasil. E-mail: magnoapr@ufla.br.

${ }^{3}$ Embrapa Arroz e Feijão (GO), UFLA, Lavras, MG, Brasil.

${ }^{4}$ Departamento de Ciência do Solo, UFLA, Lavras, MG, Brasil. 
Nos programas de melhoramento no Brasil, a avaliação das progênies e/ou linhagens é realizada sempre em presença de N. Desta forma, é questionável se seria possível a identificação de linhagens mais tolerantes ao estresse da deficiência desse nutriente nos solos (CECCARELLI, 1996; BANZIGER et al.,1997). Por outro lado, é também questionável se as linhagens existentes diferem na quantidade de grãos produzidos por $\mathrm{kg}$ de fertilizante nitrogenado aplicado.

Há alguns relatos na literatura de diferenças entre cultivares de várias espécies com relação à eficiência no uso de nitrogênio sob condições de campo, como com as culturas de milho (BANZIGER et al., 1997; AGRAMAet al., 1999; PRESTERLet al., 2003) e de trigo (GOUIS \& PLUCHARD, 1996). No caso do feijoeiro, entretanto, os relatos são escassos e envolvem um número restrito de linhagens. Além do mais, foram experimentos conduzidos já há algum tempo, com linhagens diferentes das disponíveis atualmente. Em um desses trabalhos, GUAZZELLI (1988) comparou três cultivares de feijão e verificou que elas diferiram quanto à eficiência no uso de N. As cultivares "Carioca" e "Eriparsa" foram mais eficientes que "Rio Tibagi".

Este trabalho foi conduzido visando à avaliação do desempenho de 100 linhagens de feijão, sendo a maioria com grãos tipo carioca, com o intuito de identificar aquelas com maior tolerância ao estresse de $\mathrm{N}$ e/ou também com maior resposta a esse nutriente aplicado em cobertura, que possam ser recomendadas para cultivo ou utilizadas em programas de hibridação.

\section{MATERIAL E MÉTODOS}

Os experimentos foram conduzidos em duas safras. Na safra de inverno-primavera, a semeadura ocorreu em julho de 2004 em dois locais, Lavras (sul de Minas Gerais, a 21 ${ }^{\circ} 14^{\prime} \mathrm{S}, 44^{\circ} 59^{\prime} \mathrm{W}$ e altitude média de 919m) e Lambari (sul de Minas Gerais, a 2150'S, $45^{\circ} 21^{\prime} \mathrm{W}$ e a uma altitude média de $887 \mathrm{~m}$ ). Já na safra das "águas", a semeadura foi em novembro de $2004 \mathrm{em}$

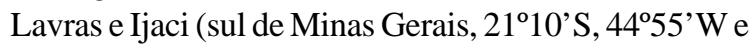
a uma altitude média de $805 \mathrm{~m}$ ).

As análises químicas dos solos nos locais em que foram conduzidos os experimentos são apresentadas na tabela 1. A semeadura foi direta, exceto em Ijaci. Para isto, procedeu-se a dessecação com glifosate cerca de 15 dias antes da semeadura.

Foram avaliadas 100 linhagens de feijão. A maioria delas (77) são provenientes do programa de melhoramento da UFLA, e algumas destas linhagens já são cultivares recomendadas. Contudo, todas elas serão denominadas de linhagens indistintamente. A maioria das linhagens possui grãos dentro do padrão carioca, isto é, grãos de cor creme com rajas marrom e peso de 100 grãos variando de 22 a $26 \mathrm{~g}$. Todas as linhagens estavam mantidas no Banco de Germoplasma da UFLA.

Em todos os locais e safras, foram conduzidos dois experimentos distintos. No primeiro, não foi efetuada adubação nitrogenada em cobertura. No segundo, foi utilizado o equivalente a $40 \mathrm{~kg} \mathrm{ha}^{-1} \mathrm{de}$

Tabela 1 - Análises químicas dos solos amostrados à profundidade de 0-20 cm, nas áreas experimentais em Lavras, Lambari e Ijaci, antes da instalação dos experimentos*.

\begin{tabular}{|c|c|c|c|c|}
\hline \multirow[b]{2}{*}{ Análise química } & \multicolumn{4}{|c|}{ Ambiente } \\
\hline & Lavras inverno 2004 & Lambari inverno 2004 & Lavras "águas" 2004/05 & Ijaci "águas" 2004/05 \\
\hline $\mathrm{pH} \mathrm{em} \mathrm{H}_{2} \mathrm{O}$ & 5,8 & 5,6 & 6,5 & 6,1 \\
\hline $\mathrm{P}\left(\mathrm{mg} \mathrm{dm}^{-3}\right)$ & 21,9 & 11,9 & 25,3 & 1,0 \\
\hline $\mathrm{K}\left(\mathrm{mg} \mathrm{dm}^{-3}\right)$ & 74,5 & 67,0 & 98,0 & 35,0 \\
\hline $\mathrm{Ca}^{2+}\left(\mathrm{cmol}_{\mathrm{c}} \mathrm{dm}^{-3}\right)$ & 2,2 & 2,4 & 3,3 & 1,8 \\
\hline $\mathrm{Mg}^{2+}\left(\mathrm{cmol}_{\mathrm{c}} \mathrm{dm}^{-3}\right)$ & 0,9 & 1,0 & 1,1 & 0,4 \\
\hline $\mathrm{Al}^{3+}\left(\mathrm{cmol}_{\mathrm{c}} \mathrm{dm}^{-3}\right)$ & 0,1 & 0,2 & 0,0 & 0,0 \\
\hline $\mathrm{H}+\mathrm{Al}\left(\mathrm{cmol}_{\mathrm{c}} \mathrm{dm}^{-3}\right)$ & 3,2 & 5,0 & 1,7 & 2,1 \\
\hline $\mathrm{SB}\left(\mathrm{cmol}_{\mathrm{c}} \mathrm{dm}^{-3}\right)$ & 3,2 & 3,6 & 4,6 & 2,3 \\
\hline $\mathrm{t}\left(\mathrm{cmol}_{\mathrm{c}} \mathrm{dm}^{-3}\right)$ & 3,3 & 3,8 & 4,6 & 2,3 \\
\hline $\mathrm{T}\left(\mathrm{cmol}_{\mathrm{c}} \mathrm{dm}^{-3}\right)$ & 6,4 & 8,6 & 6,3 & 4,4 \\
\hline $\mathrm{V}(\%)$ & 49,9 & 41,7 & 72,4 & 51,7 \\
\hline $\mathrm{m}(\%)$ & 3,0 & 5,0 & 0,0 & 0,0 \\
\hline MO (dag kg $\left.{ }^{-1}\right)$ & 1,5 & 3,6 & 4,1 & 3,1 \\
\hline P-rem $\left(\mathrm{mg} \mathrm{L}^{-1}\right)$ & 14,6 & 10,5 & 16,2 & 17,7 \\
\hline
\end{tabular}

* Análises realizadas no laboratório de análise de solos do Departamento de Ciência do Solo da UFLA (EMPRAPA, 1997).

$\mathrm{SB}=$ soma de bases; $\mathrm{t}=\mathrm{CTC}$ efetiva; $\mathrm{T}=\mathrm{CTC}$ potencial; $\mathrm{V}=$ saturação por bases; $\mathrm{m}=$ saturação por alumínio; $\mathrm{MO}=$ matéria orgânica.

Ciência Rural, v.36, n.6, nov-dez, 2006. 
$\mathrm{N}$ em cobertura, a partir do aparecimento da $2^{\mathrm{a}}$ folha trifoliolada, tendo como fonte de $\mathrm{N}$ sulfato de amônio. Em ambos os experimentos, utilizou-se o delineamento experimental látice triplo 10x10. As parcelas foram constituídas de uma linha de três metros de comprimento, espaçadas de $50 \mathrm{~cm}$, contendo 15 sementes por metro linear.

O fertilizante aplicado na semeadura, comum aos dois experimentos, foi o equivalente a $300 \mathrm{~kg} \mathrm{ha}^{-1}$ da formulação comercial 8-28-16 de N, $\mathrm{P}_{2} \mathrm{O}_{5}$ e $\mathrm{K}_{2} \mathrm{O}$, respectivamente, mais $1 \%$ de zinco presente no formulado.

Obteve-se a produtividade de grãos, colhendo-se todas as plantas da parcela, que foi submetida à análise de variância por nível dentro de cada ambiente e, posteriormente, a conjunta de todos os ambientes, considerando como fixo o efeito de níveis, os ambientes e a média. Utilizou-se o programa MSTAT - C (FREED, 1991) para realização das análises de variância. A soma de quadrado de $\mathrm{N}$ e a interação níveis $\mathrm{x}$ linhagens foram decompostas no efeito de níveis dentro de linhagens.

Utilizando os dados médios, foi estimado o índice de resposta ao nitrogênio ( $\alpha \mathrm{i})$ pela expressão de THUNG (1990), ou seja: $\alpha_{i}=\left(N_{1 i}-N_{2 i}\right) / Q$, em que: $N_{1 i}$ e $\mathrm{N}_{2 \mathrm{i}}$ : produtividade média da linhagem i na presença e ausência de nitrogênio em cobertura, respectivamente. $\mathrm{Q}$ : quantidade de $\mathrm{N}$ aplicado em cobertura. Nesse experimento, $\mathrm{Q}=40 \mathrm{~kg} \mathrm{ha}^{-1}$.

\section{RESULTADOS E DISCUSSÃO}

Em todos os ambientes (locais e safras), os experimentos com e sem $\mathrm{N}$ em cobertura foram contíguos. Esperava-se, com essa estratégia, que a diferença no comportamento das linhagens nos dois experimentos refletisse apenas a diferença no $\mathrm{N}$ aplicado, já que o manejo foi o mesmo. Ao que tudo indica, esse fato ocorreu. Esse mesmo procedimento foi adotado por BANZIGER et al. (1997), avaliando progênies de milho com e sem nitrogênio. Deve ser salientado também que a quantidade de $\mathrm{N}$ utilizada,
$24 \mathrm{~kg} \mathrm{ha}^{-1}$ de $\mathrm{N}$ na semeadura e $40 \mathrm{~kg} \mathrm{ha}^{-1}$ de $\mathrm{N}$ em cobertura, no experimento que recebeu esse nutriente, é a recomendada para a cultura do feijoeiro na região (FERREIRA et al., 2004).

$\mathrm{Na}$ análise de variância conjunta, a estimativa do coeficiente de variação experimental (CV) foi de $24,6 \%$, valor este que é semelhante ao relatado em experimentos conduzidos na região com a cultura do feijoeiro (MATOS, 2005). Considerando o número de linhagens avaliadas e a dimensão das parcelas, a precisão pode ser considerada aceitável.

Foi detectada diferença significativa $(\mathrm{P} \leq 0,01)$ para o efeito de nitrogênio. Na média das duas safras e nos locais, a produtividade obtida com $\mathrm{N}$ $\left(2487 \mathrm{~kg} \mathrm{ha}^{-1}\right)$ foi $12 \%$ acima da obtida sem $\mathrm{N}$ em cobertura $\left(2.228 \mathrm{~kg} \mathrm{ha}^{-1}\right)$ (Tabela 2$)$. A ocorrência de resposta ao nitrogênio na cultura do feijoeiro é freqüente (VIEIRA, 1998; ROSOLEM, 1996; KIKUT, 2004). VIEIRA (1998), por meio de levantamento dos experimentos de avaliação de níveis de $\mathrm{N}$ na cultura do feijoeiro, revelou que $61 \%$ dos experimentos tiveram resposta positiva à aplicação de $\mathrm{N}$.

A interação níveis $\mathrm{x}$ ambientes (safras e locais $)$ foi significativa $(\mathrm{P} \leq 0,05)$. Constatou-se resposta ao $\mathrm{N}$ em cobertura nos quatro ambientes. Contudo, a magnitude do efeito de $\mathrm{N}$ variou ligeiramente entre os ambientes, sendo menor em Lavras, no invernoprimavera, e maior em Ijaci, na safra das "águas" (Tabela 2).

A fonte de variação das linhagens foi altamente significativa $(\mathrm{P} \leq 0,01)$. Foram avaliadas linhagens, predominantemente do tipo carioca, que foram obtidas nos últimos 30 anos, no programa de melhoramento da UFLA e também de outros programas. Na média dos ambientes e dos dois níveis de $\mathrm{N}$, a linhagem com menor produtividade média foi a CII-337 $\left(1.624 \mathrm{~kg} \mathrm{ha}^{-1}\right)$, e a com maior produtividade média a Ouro Negro (3.078 $\left.\mathrm{kg} \mathrm{ha}^{-1}\right)$.

Neste trabalho, o objetivo principal foi quantificar a interação linhagens x níveis de $\mathrm{N}$. Constatou-se que essa interação foi não-significativa $(\mathrm{P} \geq 0,28)$. Considerando que o quadrado médio dessa

Tabela 2 - Produtividade média das linhagens $\left(\mathrm{kg} \mathrm{ha}^{-1}\right)$ na ausência e na presença de nitrogênio em cobertura nos quatro ambientes.

\begin{tabular}{lccc}
\hline & & Nitrogênio em cobertura & Incremento $(\%)$ \\
\cline { 2 - 4 } Ambientes & Sem & Com & 7 \\
\hline Lavras inverno-primavera & 2705 & 2894 & 5 \\
Lavras "águas" & 2248 & 2582 & 10 \\
Lambari inverno-primavera & 1881 & 2062 & 16 \\
Ijaci "águas" & 2080 & 2409 & 12 \\
Média & 2228 & 2487 & \\
\hline
\end{tabular}


interação representa a média das 4.950 interações das linhagens duas a duas, nos dois níveis (RAMALHO et al., 2005), optou-se por decompor o efeito dos níveis para cada linhagem. Nesse caso, dos 100 QM obtidos, apenas 23 deles foram significativos, realçando o que foi comentado a respeito da interação níveis $\mathrm{x}$ linhagens. Desse modo, pode-se inferir que $77 \%$ das linhagens não responderam à adubação nitrogenada em cobertura e que $23 \%$ tiveram resposta, evidenciando a possibilidade de se identificar linhagens tolerantes à ausência de $\mathrm{N}$ em cobertura e também de se identificar linhagens que respondam ao nutriente aplicado.

A relação das linhagens que responderam ao $\mathrm{N}$ aplicado em cobertura é apresentada na tabela 3. Observa-se que, das 23 linhagens com resposta ao N, em 22 delas, a resposta foi positiva. Isto é, o fertilizante nitrogenado em cobertura contribuiu para o aumento na produtividade. Já no caso da linhagem MA-I-2.5, o fertilizante em cobertura reduziu a produtividade. Em princípio, esse é um resultado não esperado. Isto pode ocorrer devido ao acaso, embora seja pouco provável, porque as médias obtidas de cada nível foram de oito repetições, isto é, quatro ambientes com duas repetições cada. Ou ainda, o maior desenvolvimento vegetativo em presença de $\mathrm{N}$ em cobertura dessa linhagem pode ter ocorrido em detrimento da parte reprodutiva. A comprovação do comportamento dessa linhagem deve ser avaliada em outros experimentos.

O desempenho médio das 22 linhagens que responderam positivamente ao $\mathrm{N}$ em cobertura foi de $29 \%$ acima do obtido sem N. Observa-se que entre essas linhagens ocorreu ampla variação na produtividade média (Tabela 3 ). Entre as 22 , das nove linhagens que produziram acima da média sem $\mathrm{N}$ $\left(1.941 \mathrm{~kg} \mathrm{ha}^{-1}\right)$, oito delas estão entre as nove com maior produtividade e com maior resposta positiva ao $\mathrm{N}$.

O que se procura também são linhagens que respondam ao investimento em fertilizantes, produzindo maior quantidade de grãos por $\mathrm{kg}$ de fertilizante utilizado. Entre as linhagens com resposta positiva, o índice de eficiência proposto por THUNG (1990) variou de 11,3 a 18,3, ou seja, o mínimo de resposta foi $11,3 \mathrm{~kg}$ de grãos por $\mathrm{kg}$ de $\mathrm{N}$ aplicado em cobertura (CI-257) a 18,3kg de grãos por kg de N (RC-I-3).

Reposta diferencial de cultivares (linhagens ou híbridos) tem sido constatada, em algumas oportunidades, na cultura do milho. BANZIGER et al. (1997) avaliaram progênies de Irmãos Germanos e endogâmicas $\mathrm{S}_{1}$ e $\mathrm{S}_{2}$ obtidas pelo CIMMYT, no período de 1986 a 1995, em presença e ausência de N. Esses autores observaram diferença no desempenho das progênies com e sem N. Evidenciaram que, se o objetivo é obter linhagens para as condições de baixo N, comuns nas regiões tropicais, a seleção deve ser realizada nessa condição para se aumentar a chance de sucesso com a seleção. Resultados semelhantes foram relatados por MURULI \& PAULSEN (1981). Em trigo, GOUIS \& PLUCHARD (1996) avaliaram 26 linhagens e identificaram diferença expressiva na tolerância. Esses mesmos autores constataram que os híbridos de trigo mostraram maior heterose sob condições de estresse de N.

Devido à importância sócio-econômica e ambiental da cultura do feijoeiro, trabalhos como esses deveriam receber maior ênfase. Seria importante avaliar essas linhagens em condições de maior estresse de $\mathrm{N}$, ou seja, sem o emprego desse nutriente na semeadura e cobertura.

\section{CONCLUSÕES}

A produtividade de grãos com $\mathrm{N}$ em cobertura foi $12 \%$ superior à obtida sem N.

Tabela 3 - Produtividade média das linhagens responsivas à aplicação de nitrogênio, na presença e na ausência do nutriente, com seus respectivos índices de eficiência de utilização de nitrogênio $(\alpha)$.

\begin{tabular}{lccc}
\hline & \multicolumn{3}{c}{ Produtividade em kg ha } \\
Linhagens & Sem N & Com N & $\alpha$ \\
\hline CI-140 & 1517 & 2021 & 12,6 \\
CI-257 & 1744 & 2195 & 11,3 \\
CII-244 & 1994 & 2472 & 11,9 \\
CII-90 & 1915 & 2420 & 12,6 \\
ESAL 507 & 1761 & 2338 & 14,4 \\
ESAL 629 & 1824 & 2446 & 15,6 \\
ESAL 1 & 1853 & 2385 & 13,3 \\
H-15 & 1741 & 2220 & 11,9 \\
H-4 & 1760 & 2249 & 12,2 \\
H-87 & 1534 & 2084 & 13,3 \\
H-92 & 1498 & 2000 & 12,5 \\
MA-I-6.10 & 1908 & 2530 & 15,6 \\
LH-11 & 1938 & 2479 & 13,5 \\
CNFC 8063 & 2465 & 3102 & 15,9 \\
Z-22 & 2292 & 2890 & 14,9 \\
Z-6 & 2348 & 2842 & 12,3 \\
CV-55 & 2209 & 2792 & 14,6 \\
CV-46 & 2309 & 2950 & 16,0 \\
RC-I-7 & 1839 & 2368 & 13,2 \\
RC-I-3 & 2272 & 3003 & 18,3 \\
AN-LAV-51 & 2025 & 2725 & 17,5 \\
CIII-H-4-12 & 1961 & 2574 & 15,3 \\
\hline Média & 1941 & 2504 & \\
\hline MA-I-2.5 & 3115 & 2340 & $-19,4$ \\
\hline Média das 77 linhagens & 2299 & 2483 & \\
sem resposta ao N & & \\
\hline & & &
\end{tabular}


Apenas 22 linhagens, das 100 testadas, responderam positivamente à aplicação de $\mathrm{N}$ em cobertura. Para essas linhagens, a eficiência na utilização do nutriente variou de 11,3 a 18,3kg de grãos por kg de $\mathrm{N}$ aplicado.

\section{AGRADECIMENTOS}

Nossos sinceros agradecimentos ao Conselho Nacional de Desenvolvimento Científico e Tecnológico (CNPq), pela bolsa de iniciação científica concedida; e à FAPEMIG, pelo financiamento do projeto de pesquisa.

\section{REFERÊNCIAS}

AGRAMA, H.A.S. et al. Identification of quantitative trait loci for nitrogen use efficiency in maize. Molecular Breeding, v.5, p.187-195, 1999.

ALVES, V.G. Resposta do feijoeiro a doses de nitrogênio no plantio e cobertura e à inoculação de sementes com rizóbio. 2002. 46f. Dissertação (Mestrado em Fitotecnia) Curso de Pós-graduação em Agronomia, Universidade Federal de Lavras

BANZIGER, M. et al. Efficiency of high-nitrogen selection environments for improving maize for low-nitrogen target environments. Crop Science, Madson, v.37, n.4, p.11031109,1997 .

CECCARELLI, S. Adaptation to low/high input cultivation. Euphytica, v.92, p.203-214, 1996.

EMPRESA BRASILEIRA DE PESQUISA AGROPECUÁRIA EMBRAPA- Centro Nacional de Pesquisa de Solos. Manual de métodos de análise de solo. 2.ed. Rio de Janeiro, 1997. $212 \mathrm{p}$.

FERREIRA, A.C.B. et al. Feijão de alta produtividade. Informe Agropecuário, Belo Horizonte, v.25, n.223, p.61-72, 2004.

FREED, R.D. MSTAT - C. A software program for the design, management and analysis of agronomic research experiments. East Lansing: Michigan State University, 1991. p.irr.

GOUIS, J.L.; PLUCHARD, P. Genetic variation for nitrogen use efficiency in winter wheat (Triticum aestivum L.). Euphytica, v.92, p.221-224, 1996.
GUAZZELLI, E.M.F.M. Efeito de nitrato e amônio no crescimento, assimilação e eficiência de utilização do nitrogênio por cultivares de feijoeiro (Phaseolus vulgaris L.) na fase inicial de crescimento. 1988. 112f. Dissertação (Mestrado em Solos e Nutrição de Plantas) - Curso de Pósgraduação em Agronomia, Universidade Federal de Lavras.

KIKUT, H. Resposta do feijoeiro (cv. BRS-MG Talismã) a doses de nitrogênio e fósforo. 2004. 124f. Tese (Doutorado em Fitotecnia) - Curso de Pós-graduação em Agronomia, Universidade Federal de Lavras.

MATOS, J.W. de. Análise crítica do programa de melhoramento genético do feijoeiro da UFLA no período de 1974 a 2004. 2005. 116f. Tese (Doutorado em Genética e Melhoramento de Plantas) - Curso de Pós-graduação em Agronomia, Universidade Federal de Lavras.

MURULI, B.I.; PAULSEN, G.M. Improvement of nitrogen use efficiency and its realtionship to other traits in maize. Maydica, v.26, p.63-73, 1981.

PRESTERL, T. et al. Improvement nitrogen-use efficiency in European maize: Estimation of quantitative genetic parameters. Crop Science, v.43, n.4, p.1259-1265, 2003.

RAMALHO, M.A.P. et al. Experimentação em genética e melhoramento de plantas. 2.ed. Lavras: UFLA, 2005. 300p.

ROSOLEM, C.A. Calagem e adubação mineral. In: ARAÚJO, R.S. et al. Cultura do feijoeiro comum no Brasil. Piracicaba: POTAFOS, 1996. p.353-390.

SILVA, M.V. da. Aplicação foliar de cobalto, inoculação com rizóbio e adubação nitrogenda na cultura do feijoeiro. 2002. 54f. Dissertação (Mestrado em Fitotecnia) - Curso de Pós-graduação em Agronomia, Universidade Federal de Lavras.

THUNG, M. Phosporus: a limiting nutrient in bean (Phaseolus vulgaris L.) production in Latin America and field screening for efficiency and response. In: El BASSAM et al. (Ed). Genetic aspects of plant mineral nutrition. Dordrecht, Netherlands: Kluwer, 1990. p.501-521.

VIEIRA, C. Adubação mineral e calagem. In: VIEIRA, C. et al. (Ed). Feijão: aspectos gerais e cultura do Estado de Minas. Viçosa: UFV, 1998. p.123-152. 